\title{
Development of the Los Alamos continuous high average-power microsecond pulser ion accelerator
}

\author{
L. J. Bitteker, B. P. Wood, H. A. Davis, and W. J. Waganaar \\ Plasma Physics Group, Los Alamos National Laboratory, Los Alamos, New Mexico 87545 \\ I. D. Boyd \\ University of Michigan, Ann Arbor, Michigan 48109
}

R. H. Lovberg

University of California, San Diego, California 92093

(Received 24 April 2000; accepted for publication 24 July 2000)

\begin{abstract}
The continuous high average-power microsecond pulser (CHAMP) ion accelerator is being constructed at Los Alamos National Laboratory. Progress on the testing of the CHAMP diode is discussed. A direct simulation Monte Carlo computer code is used to investigate the puffed gas fill of the CHAMP anode. High plenum pressures and low plenum volumes are found to be desirable for effective gas puffs. The typical gas fill time is $150-180 \mu$ s from initiation of valve operation to end of fill. Results of anode plasma production at three stages of development are discussed. Plasma properties are monitored with electric and magnetic field probes. From this data, the near coil plasma density under nominal conditions is found to be on the order of $1 \times 10^{16} \mathrm{~cm}^{-3}$. Large error is associated with this calculation due to inconsistencies between tests and the limitations of the instrumentation used. The diode insulating magnetic field is observed to result in lower density plasma with a more diffuse structure than for the cases when the insulating field is not applied. The importance of these differences in plasma quality on the beam production is yet to be determined. (C) 2000 American Institute of Physics. [S0034-6748(00)06010-X]
\end{abstract}

\section{INTRODUCTION}

The continuous high average-power microsecond pulser (CHAMP) high-intensity ion accelerator is being developed at Los Alamos National Laboratory. A detailed description of the design and the motivation for the development of the CHAMP accelerator is discussed elsewhere. ${ }^{1-3}$ For a general discussion of high-intensity ion beams and diodes, see Ref. 4. Briefly, CHAMP utilizes a magnetically insulated diode with a plasma anode designed to produce a repetitively pulsed ion beam as shown in Fig. 1. The plasma beam is produced by the following series of timed events. First, the slow insulating magnetic field with a rise time of $500 \mu$ s is applied parallel to the induction coil surface. Next, a puff of gas is injected radially over the induction coil surface as discussed in the next section. The anode plasma is produced by four parallel, two-turn induction coils pulsed to a potential of $30 \mathrm{kV}$ and current of several thousand amperes with a rise time of $1 \mu \mathrm{s}$. The $\mathbf{j}_{\theta} \times \mathbf{B}_{r}$ force produced by the plasma current and the magnetic field of the induction coils pushes the anode plasma against the insulating magnetic field at the entrance to the anode-cathode gap where the ions are extracted. Finally, four parallel Blumlein transmission lines switched through thyratrons for repetitive operation, or spark gaps for single shot operation, raise the potential of the anode structure to $+250 \mathrm{kV}$. The high potential accelerates the anode plasma ions through the insulating gap and past the cathode towards a ballistic focus. The plasma electrons remain trapped on the insulating field lines in the anodecathode gap due to their smaller mass while the ions are accelerated with minimal deflection from the magnetic field. As the plasma ions pass between the cathode cones, fieldemitted electrons are entrained, neutralizing the ion space charge downstream of the gap. Electrons trapped in the anode-cathode gap allow for ion current densities well above the space charge limit for a vacuum gap. The CHAMP accelerator is designed to produce an ion beam with a potential of $200-250 \mathrm{kV}$, ion current of $15 \mathrm{kA}$, pulse duration of $1 \mu \mathrm{s}$, and pulse frequency up to $30 \mathrm{~Hz}$.

Later we discuss the progress of the accelerator development and report on the results of two completed steps. Specifically, the simulation of the gas fill process and the initial production of the anode plasma are described. The assembly and testing of the accelerator has made significant progress. Several subsystems have been assembled and tested including the accelerator diode, vacuum system, cathode, puff valve, and insulating magnetic field coils. Preliminary versions of the induction coil driver circuit, insulating field circuit, and gas puff valve controller are also operating. Finally, a single shot version of the Blumlein transmission lines using a spark gap in place of the thyratrons has been assembled and is being tested for initial beam production experiments.

\section{GAS FILL SIMULATION}

To produce an ion beam from a plasma diode, a series of well-timed events must occur as described in the previous section. Clearly, the gas fill process directly affects the timing and quality of the production of the anode plasma. The quality of the plasma refers to the degree of ionization and 


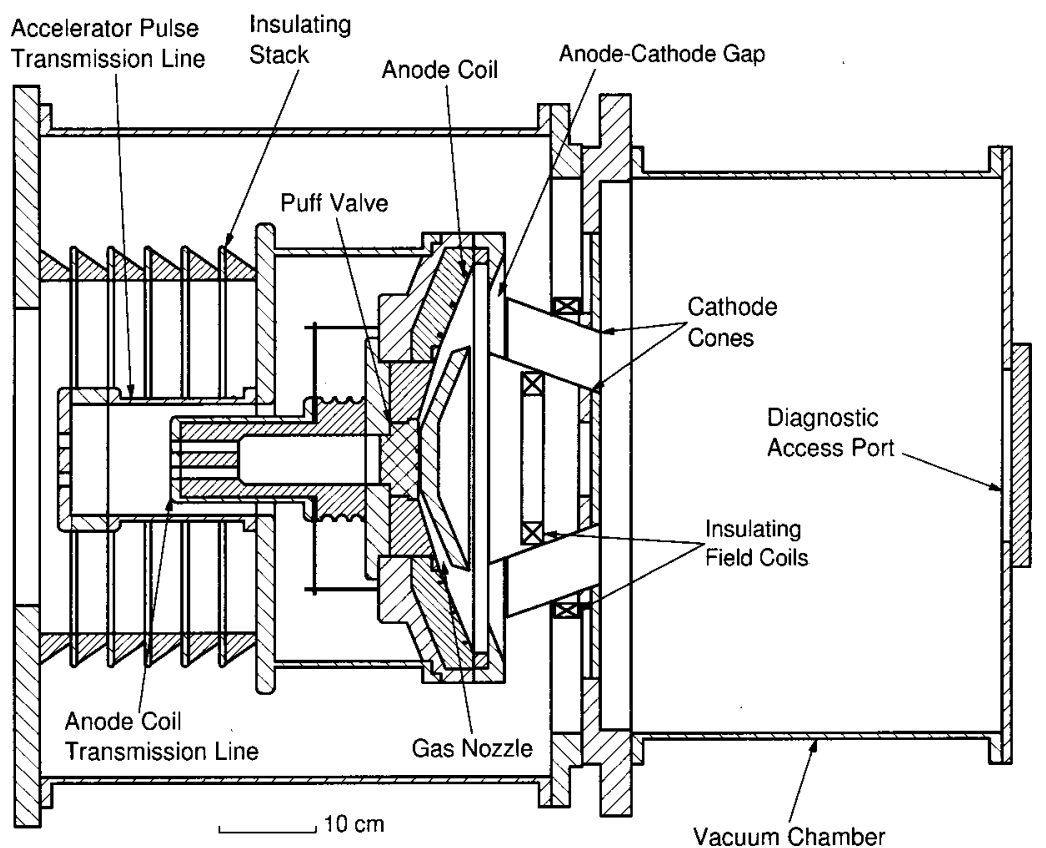

FIG. 1. Layout of the CHAMP plasma diode for testing. Left side of assembly attaches to an insulating oil tank housing the puff valve controller, induction coil pulse circuit, and accelerator pulse transmission lines (not shown). the uniformity of the resulting plasma ring. Ideally, the gas uniformly covers the surface in front of the induction coils to approximately $100 \mathrm{mTorr}$ with no leakage into the anodecathode gap. At the moment this condition is achieved, the induction coils are pulsed, producing a uniform anode plasma with minimal spoiling of the vacuum from excess gas. In practice, it is not possible to introduce the gas onto the surface of the coils without density gradients and some leakage into the gap. Therefore, the dynamic behavior of the fill process must be examined in detail to optimize the gas nozzle geometry, the puff valve operating parameters, and the induction coil pulse timing.

An Applied Pulsed Power, Inc., fast acting puff valve utilizing a metallic diaphragm actuated by eddy currents is used on CHAMP as shown in Fig. 2. The typical opening

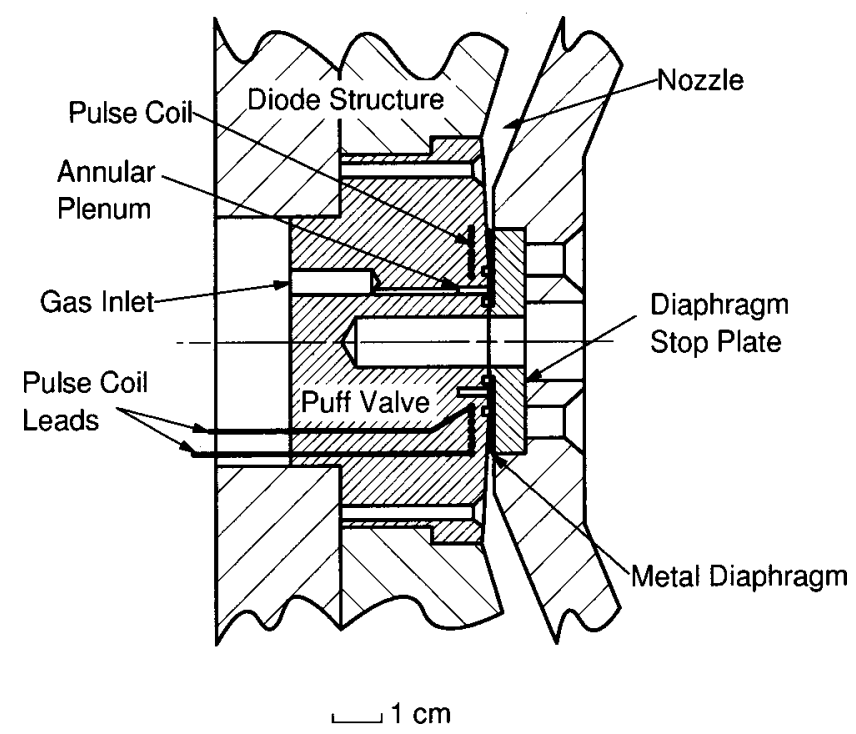

FIG. 2. Schematic diagram of the puff valve. time of the valve is $125 \mu$ s and full deflection of the edge of the diaphragm is $0.5 \mathrm{~mm}$. The puff valve has an integral gas plenum behind the diaphragm that includes gas feed ports leading into a machined annular grove. Since the feed ports are always open, the effective plenum volume associated with the feeds is a function of the open to close time of the diaphragm. For the valve currently used on CHAMP, the effective plenum volume in these simulations is approximately $0.25 \mathrm{~cm}^{3}$. This volume is calculated from the geometry, the opening time, and the plenum pressure.

The gas plenum volume and pressure are two parameters of the puff valve that can be controlled to achieve the desired gas fill. In principle, the volume of this plenum could be controlled by changing the dimensions of the annular plenum, changing the diameter of the feed ports, and including valves on the feed lines. Effects due to changes in the plenum volume are modeled as described later; however, no experiments on the CHAMP accelerator using other puff valve designs have been performed. The design of the puff valve is not exactly azimuthally symmetric due to the finite number and placement of the feed ports. For the model of puff valve used in these experiments, the valve has one gas inlet port located at the bottom of the annular plenum as shown in Fig. 2. This asymmetry is not considered in the simulations and is not expected to be significant early in the fill. Neglecting this asymmetry is justified for the large plenum used in the experiments because only a small fraction of the gas early in the fill is used in the plasma production as discussed below.

The valve and nozzle geometry is shown in Fig. 3. The target pressure for the gas fill is on the order of 100 mTorr and the typical dimension in the nozzle is $1 \mathrm{~cm}$. The ratio of the characteristic mean free path to the length scale, or Knudsen number, for this target condition is 0.04 . This Knudsen number corresponds to flow in the transition region between molecular flow and compressible fluid flow. Since 


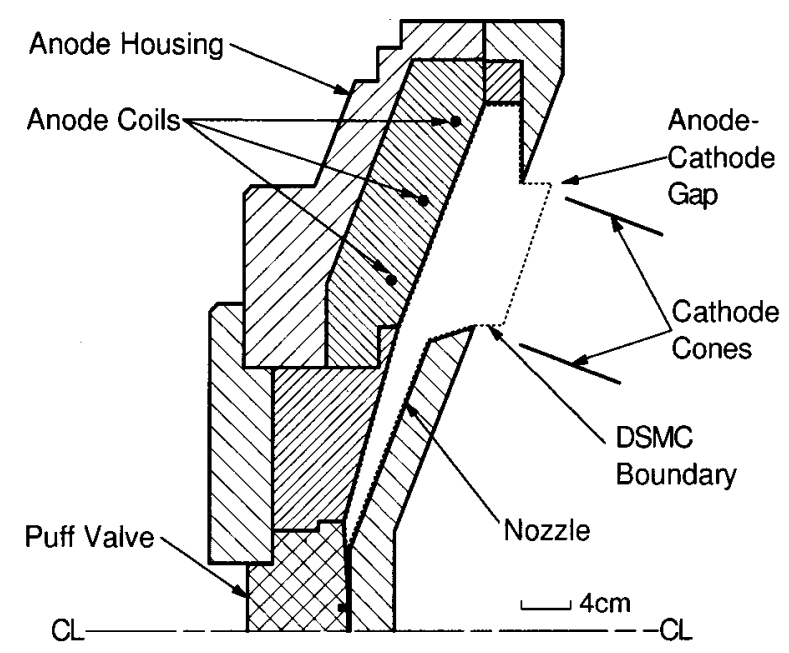

FIG. 3. Close-up sectional view of the nozzle and valve layout showing the boundary of the DSMC computer model calculations (dotted line).

molecular motion is non-negligible, the Navier-Stokes equations are invalid and a kinetic model must be used.

A computer simulation of the fill process in CHAMP was used in this analysis employing a FORTRAN 77 direct simulation Monte Carlo (DSMC) code. ${ }^{5,6}$ This code models two-dimensional or axisymmetric nonequilibrium gas flow and includes features for handling multiple species, unsteady flow, relaxation of the rotational energy mode of molecules, and diffuse reflection off of solid surfaces. The energy spectrum of the gas particles is also tracked. A set of operational parameters is provided to the code in an input file. These parameters include the number and types of gases; initial conditions for temperature, density, and velocity; as well as various parameters that control the execution of the code. The geometry of the CHAMP gas nozzle, shown in Fig. 3, is defined in a series of data statements in a separate routine that creates the grid used in the main simulation code.

For these simulations, the puff valve was assumed to open instantaneously. The gas fill simulation began with the gas molecules, in this case hydrogen, crossing a surface axially symmetric about the system axis a few millimeters from the valve opening where the nozzle begins to diverge. For each run, two-dimensional maps of time-averaged particle properties were produced in $8 \mu$ s steps for a minimum total simulation time of $80 \mu \mathrm{s}$. In these analyses, the properties of interest were the particle number density and pressure. The objective of the simulations was to determine the conditions required for the gas fill to be localized over the anode coil surface at a pressure on the order of 100 mTorr with minimal leakage into the anode-cathode gap. Although the density is the controlling parameter for breakdown of the gas, the pressure is easier to measure in practice and is therefore included in this analysis. Four cases were simulated as summarized in Table I. Cases 1-3 examined the influence of plenum pressure and volume on the gas fill. Case 4 simulated the gas fill with the geometry of the nozzle updated to include minor changes in the nozzle shape required during assembly and testing of the anode for comparison to experiments.

Four plots from case 1 of the calculated pressure as a function of position at times of $24,40,56$, and $72 \mu$ s are
TABLE I. Summary of plenum conditions used in the simulations. Cases 1 and 4 differ only in details of the geometry.

\begin{tabular}{ccc}
\hline \hline Case & Plenum pressure (psia) & Plenum volume $\left(\mathrm{cm}^{3}\right)$ \\
\hline 1,4 & 30 & 0.25 \\
2 & 3 & 0.25 \\
3 & 30 & 0.025 \\
\hline \hline
\end{tabular}

presented in Fig. 4. Refer to Fig. 3 for a description of the simulation boundary. As listed in Table I, the plenum pressure in case 1 is 30 psia and the plenum volume is $0.25 \mathrm{~cm}^{3}$. The labeled times correspond to the time since the beginning of the simulation and do not take into account the time for the valve to open. The $10 \mathrm{mTorr}$ isobar crosses the nozzle outlet in $24 \mu \mathrm{s}$. The flow continues to expand over the coil surface primarily in the radial direction and to a lesser extent in the axial direction. At approximately $40 \mu \mathrm{s}$, the pressure of the gas reflecting off of the outer wall of the anode reaches 10 mTorr. The desired fill conditions are achieved between 56 and $72 \mu$ s. At $56 \mu$ s, the pressure across the anode opening to the anode-cathode gap is on the order of 10 mTorr and the pressure along the surface of the coils ranges from approximately $50-200 \mathrm{mTorr}$. The isobars on the order of a few Torr show little movement during the duration of the simulation and never leave the nozzle region. By $72 \mu \mathrm{s}$, the
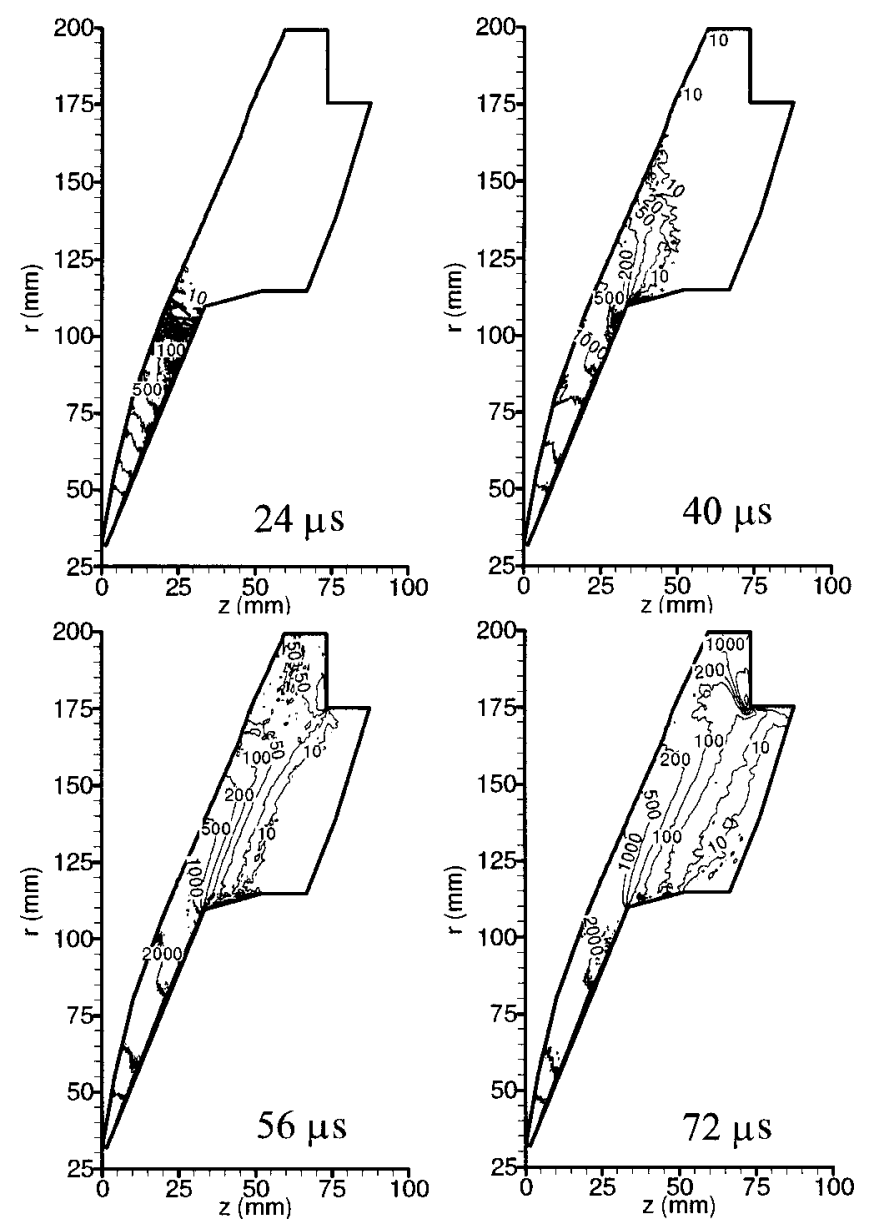

FIG. 4. Plots of pressure as a function of position for the gas flow simulation in case 1 at 24, 40, 56, and $72 \mu \mathrm{s}$. Units are mTorr. 

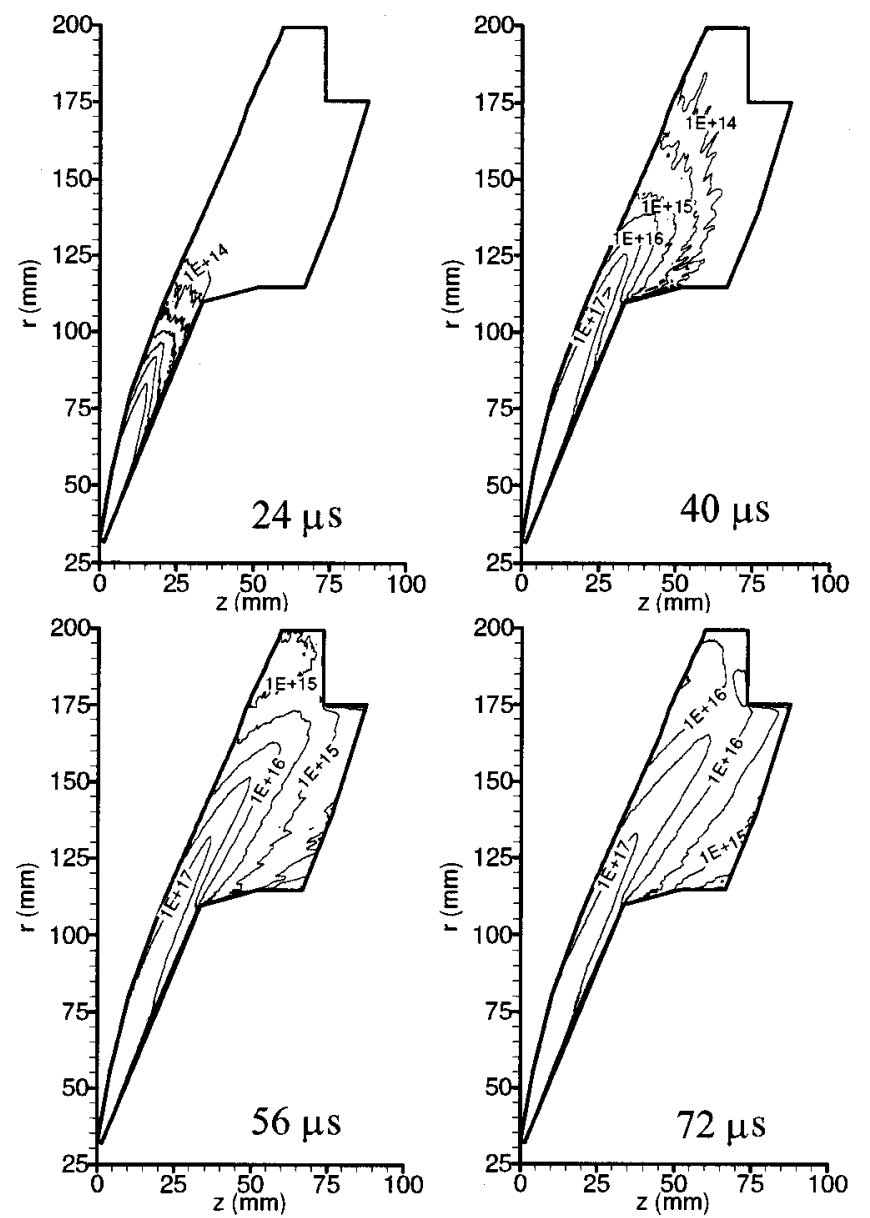

FIG. 5. Plots of particle number density as a function of position for the gas flow simulation in case 1 at $24,40,56$, and $72 \mu$ s. Units are particles $/ \mathrm{cm}^{3}$.

$500 \mathrm{mTorr}$ isobar is beginning to move across the coil surface and the 2000 mTorr isobar shows movement that is more significant. The existence of the high pressure isobars upstream at the time of desired fill conditions indicates a significant quantity of extra gas is released into the system, which would have to be evacuated prior to the next beam being produced. More importantly, at the end of the simulation the $10 \mathrm{mTorr}$ isobar has moved approximately $2 \mathrm{~cm}$ into the gap, which could result in a discharge between the anode and cathode.

In general, the flow exhibits viscous interaction with the wall. This is clearly seen in the density plots shown in Fig. 5 by the development of a nozzle centered neutral gas beam forming within a large boundary layer. The flow separates from the coil sidewall beginning around $56 \mu$ s as the flow expands into the gap. The separation becomes more dramatic as gas bubbles stagnate in the corners of the outer surface of the anode at $72 \mu \mathrm{s}$. By the end of the simulation, the central gas beam is deflected directly towards the outer edge of the gap opening. When the desired fill conditions are achieved between 56 and $72 \mu \mathrm{s}$, the density at the surface of the coils ranges from $10^{15}$ to $10^{16} \mathrm{~cm}^{-3}$.

The objective of cases 2 and 3 is to minimize the amount of gas introduced into the vacuum while maintaining the desired fill characteristics demonstrated in case 1 . In case 2 the plenum pressure is reduced to 3 psia while the plenum volume is left at $0.25 \mathrm{~cm}^{3}$. The reduction in plenum pressure results in the flow requiring significantly longer time to develop relative to case 1 . The simulation in case 2 requires a total simulation time of $112 \mu$ s to accommodate this slower development. The flow exits the nozzle at approximately 40 $\mu \mathrm{s}$ as compared to $24 \mu \mathrm{s}$ in case 1 . The maximum radial travel of the 100 mTorr isobar is approximately $135 \mathrm{~mm}$, which only slightly overlaps the coils. By comparison, in case 1 the $100 \mathrm{mTorr}$ isobar extends to about $170 \mathrm{~mm}$. The maximum pressure isobar seen in the case 2 simulation is greatly reduced to $2000 \mathrm{mTorr}$ compared to $10000 \mathrm{mTorr}$ in case 1 indicating a significant reduction in the amount of excess gas released.

In case 2, the boundary layer is thicker, accounting for the slower flow development. The lower plenum pressure results in a gas beam with lower radial momentum so it does not have sufficient energy to effectively overcome the frictional losses in the nozzle. Since the axial momentum component of the flow is proportionately larger compared to the radial momentum in this case, the flow expands more readily into the gas before the surface pressure reaches the desired level. Although the lower plenum pressure does reduce the quantity of fill gas released into the system, it also results in a less effective fill.

In case 3 the plenum pressure is set to the original value of 30 psia while the plenum volume is reduced by an order of magnitude to $0.025 \mathrm{~cm}^{3}$. This configuration results in fill characteristics similar to case 1 while significantly reducing the pressure upstream in the nozzle. The gas fill is slightly slower than in case 1 with the optimum conditions being achieved around $72 \mu \mathrm{s}$. The $100 \mathrm{mTorr}$ isobar reaches a maximum radial position of $150 \mathrm{~mm}$ which is over the coils. Leakage into the gap is significantly reduced. The $10 \mathrm{mTorr}$ isobar extends less than $1 \mathrm{~cm}$ into the gap at $80 \mu$ s compared to $2 \mathrm{~cm}$ for case 1 . The upstream pressure in the nozzle decreases throughout the simulation, which is a feature not seen in either of the earlier cases, indicating a more efficient use of the fill gas. The gas beam behavior is similar to that in case 1. Of the cases considered, the parameters used in case 3 produce the best gas fill.

Case 4 uses the same plenum conditions as case 1 with minor geometric modifications to better match the real CHAMP nozzle currently in use. Most notably, the real nozzle has a more pronounced divergence close to the puff valve exit. Although there are some changes in the details of the simulated flow behavior between the two cases, the overall behavior is very similar. Therefore, the results of cases 1-3 are reasonable for predicting the flow behavior in the actual CHAMP geometry.

Although not an exhaustive analysis of the parameter space, several generalized conclusions can be made based on these results. First, the plenum volume should be optimized for the desired fill conditions, especially for operation in a rep-rate mode, by minimizing its volume. Second, the plenum pressure should be relatively high to minimize early axial expansion and provide the energy necessary to push the desired isobar over the coil face. The nozzle geometry should be designed to provide the minimal rate of divergence, especially near the nozzle outlet, in order to maximize 
the radial gas beam momentum while minimizing the axial momentum.

Since minimal attempt was made to optimize the design of the nozzle and fill volume geometry prior to fabrication, a couple of options for more extensive modification may be beneficial. None of these modifications have been analyzed in detail. They are mentioned here as potential future investigations. Based on the fill behavior observed in these simulations, it may be desirable to design the outer edge of the fill volume such that the neutral gas beam reflects radially back on itself rather than being deflected in the axial direction. Another option is to remove the outer wall completely and apply differential pumping at the outer radius. This second option might provide a means for allowing a continuous stream of gas to flow over the anode face that could be ionized, as needed at the rate the rest of the system operates. Certainly many other designs are possible.

\section{INITIAL ANODE PLASMA PRODUCTION EXPERIMENTS}

The testing of the anode plasma proceeded in three stages. First, the plasma was produced using a static gas fill to determine the range of pressure and coil potential that effectively ionized the gas. Second, plasma was produced from a puffed gas fill without the insulating magnetic field applied. Finally, plasma was produced from a puffed gas fill with the insulating magnetic field applied and the cathode structure installed. In all three stages, the plasma conditions were measured with a magnetic coil $(\dot{B})$ probe and a floating differential electric field $(E)$ probe. Both probes were mounted on a positioning stage with four directions of motion and oriented to measure only one field component at a time. In addition to the field probes, a framing camera and a photodiode sensor were used to qualitatively characterize the plasma.

\section{A. Probe design and application}

The theory, design, and application of the field probes used here are briefly described later. See Ref. 7 for a detailed discussion of these types of probes. The primary objective of these experiments is to measure the plasma density. The electron density can be calculated using the generalized form of Ohm's Law ${ }^{8}$

$$
\mathbf{E}+\mathbf{v} \times \mathbf{B}-\frac{1}{n_{e} e}\left(\mathbf{J} \times \mathbf{B}-\nabla p_{e}\right)-\eta \mathbf{J}=0,
$$

where $\mathbf{v}$ is the charge velocity, $n_{e}$ is the electron number density, $\mathbf{J}$ is the current density, $p_{e}$ is the electron pressure, and $\eta$ is the electron resistivity. For application to the plasma diode, cylindrical coordinates are used. For these experiments, we limit the analysis to times when the electron pressure gradient is much less than the $\mathbf{J} \times \mathbf{B}$ term. For the CHAMP anode, $\mathbf{v}$ is approximately axial, and hence, the $\mathbf{v}$ $\times \mathbf{B}$ term has no $z$ component. Similarly, the current density, $\mathbf{J}$, is purely azimuthal and therefore does not contribute to the $z$ component. Focusing on the $z$ component and rearranging terms, Ohm's Law reduces to

$$
n_{e}=\frac{J_{\theta} B_{r}}{e E_{z}} .
$$

From Maxwell's Equations, the azimuthal current is related to the radial magnetic field by

$$
J_{\theta}=\frac{1}{\mu_{0}} \frac{\partial B_{r}}{\partial z} .
$$

From (2) and (3), and combining the $B_{r}$ terms into the derivative of the square of $B_{r}$ provides the desired form of the expression for the electron density

$$
n_{e}=\frac{1}{2 \mu_{0} e E_{z}} \frac{\partial\left(B_{r}^{2}\right)}{\partial z}
$$

Therefore, from the measurement of the radial magnetic field and the axial electric field as a function of axial position, the electron density can be calculated. In these experiments, the plasma is assumed to be charge neutral prior to the beam accelerating pulse so the electron density is equal to the ion density times the ionization level. For the calculation of electron density from (4) and experimental data, a central difference approximation of the derivative term and the average of the electric field in the interval are used.

By collecting magnetic and electric field data at various axial positions, with reasonable repeatability between tests, the density of the plasma sheets is determined. For these experiments, data is collected every $5 \mathrm{~mm}$ along the $z$ axis. Due to the large spatial interval between data points, the approximation of the derivative term in (4) is rough. Furthermore, only one probe is used at a time and the shot-to-shot repeatability is low, further reducing accuracy in the calculations. Inconsistent behavior is particularly apparent in the $E$ probe data. A series of more detailed experiments using multiple probes simultaneously is necessary to determine the plasma density more accurately; however, these preliminary data sets are sufficient to make an estimate of the plasma density.

In addition to calculating the electron density, the magnetic field probe is used to monitor the plasma current sheets moving axially away from the anode coils. The $\dot{B}$ probe records the magnetic field produced by both the ringing current in the anode coils and the current flowing in the plasma. Without the plasma current, the signal from the anode coil current is a decaying sinusoid. As the plasma sheets pass over the probe, the probe signal is distorted by the presence of plasma currents as shown in the sample $\dot{B}$ probe signal in Fig. 6. The distortion is due to the shielding of the anode coil field by the plasma current field. The distortion magnitude, abruptness, and duration are used to qualitatively evaluate the plasma current sheets. Large reversal of field indicates large plasma current while abrupt and short duration distortions indicate a well-formed sheet. This qualitative information is useful for detecting cases in which weak plasma currents are observed corresponding to low plasma density. By comparing a series of magnetic field plots at different axial positions, the velocity of the plasma sheets can be calculated.

The $\dot{B}$ probe used for these experiments consists of a total of 21 turns of 30 ANG magnet wire in two layers with 


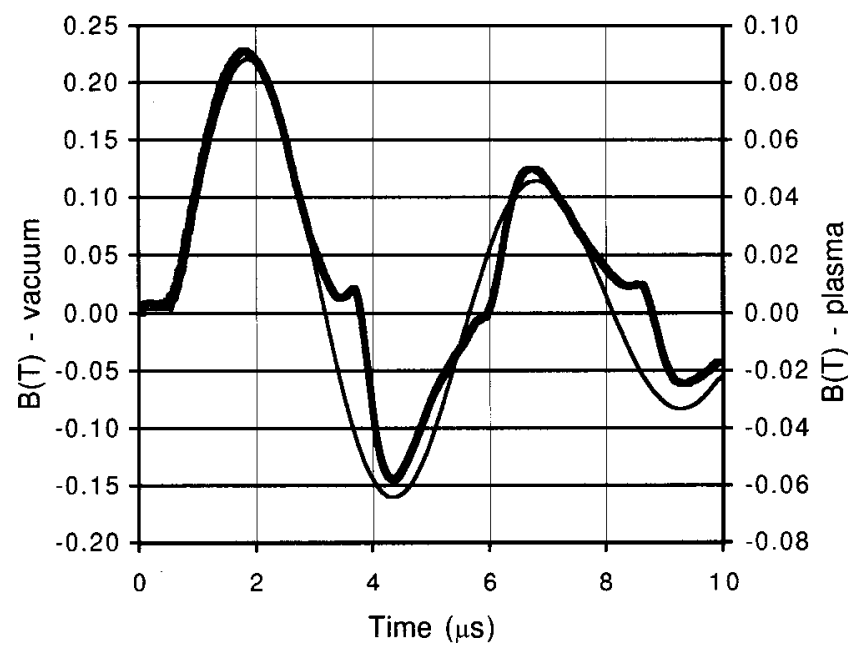

FIG. 6. Sample plot of the magnetic field as a function of time recorded using the $\dot{B}$ probe. The fine line plot is the magnetic field in vacuum used for reference. Deviations of the field in plasma (heavy line) from the form of the vacuum field indicate plasma effects.

an effective diameter of $2 \mathrm{~mm}$ and length of $2 \mathrm{~mm}$. The coils are wound on an acrylic form and encased in epoxy resin. The twisted leads from the coil are connected to a RC integrating circuit with a time constant of $47.6 \mu \mathrm{s}$. The output of the integrator is recorded on an oscilloscope. Calibration of the coil is accomplished by comparison to a larger probe with easily measured geometry in a common field. The probe sensitivity is measured to be $1.32 \mathrm{~V} / \mathrm{T}$.

The $E$ probe is constructed from a section of $1 / 4$ in. rigid coaxial cable with the center conductor and insulation extending $8 \mathrm{~mm}$ past the outer conductor. To eliminate the common mode, the leads of the $E$ probe are connected to a shielded differential transformer. This transformer is constructed from a ferrite core with outer diameter of 1.5 in., inner diameter of 1 in., and thickness of 3/8 in. To minimize coil-to-coil capacitive coupling through the core, the core is encased in a copper shield with a circumferential slit to allow flux entry. Both the primary and secondary windings consist of ten turns, with the primary wire insulated for high voltage. A copper electrostatic shield is placed between the primary and secondary coils and attached to the core shield to minimize line-of-sight capacitive coupling between the coils. The entire core assembly is enclosed in an aluminum box with BNC connectors on both ends. An additional $430 \Omega$ resistance in series with the $50 \Omega$ output cable is added to the output to minimize the rise time, which is limited by leakage inductance. To prevent significant differentiation of the signal due to loading of the primary coil inductance, the source must be of relatively low impedance. For the pulsed plasma experiments considered here, differentiation is negligible because the source impedance is on the order of $<1 \Omega$ resulting in a time constant of $300 \mu \mathrm{s}$, which is much longer than the pulse length. The measured inductance of the transformer primary is $300 \mu \mathrm{H}$. Common-mode reduction is calculated to be $99.7 \%$. The ratio of input to output signal is measured to be 0.105 .

In addition to the $E$ and $\dot{B}$ probes, a simple photodiode is used to qualitatively monitor the plasma. Specifically, the photodiode provides a means for observing the plasma glow due to recombination. Though not a quantitative instrument, the photodiode has proven very useful in comparing the shotto-shot behavior of the plasma. It is also valuable in determining the onset of breakdown in the fill gas and subsequent ionization peaks due to the ringing of the anode coils.

\section{B. Experimental results}

In the first experiments to produce plasma, a static fill of various gases was ionized to examine the uniformity of the anode plasma and the range of presusre and coil potential at which various species breakdown. Although the density is the significant gas property in causing breakdown, the pressure was used since it is much easier to measure. All experiments were conducted at ambient temperature so the density and pressure are linear functions of each other. In general, these experiments were intended to provide qualitative data so no plasma density calculations are reported here. Axial uniformity of the anode was examined with the use of a framing camera set up to photograph the entire anode at $1 \mu \mathrm{s}$ intervals for a total of $8 \mu \mathrm{s}$. Acceptable uniformity was observed with some filamentation apparent.

The second set of experiments involved the production of the anode plasma from a puffed hydrogen gas fill without applying the insulating magnetic field. The objective of these experiments was to determine the nominal timing of the gas puff and the anode coil pulse using the earlier puff fill simulations as a guide. The effectiveness of the plasma production as a function of the event timing was evaluated with the $\dot{B}$ probe and the photodiode. A delay of $150-180 \mu$ s between triggering the puff valve and the anode coil pulses was found to be optimum. As discussed earlier, the simulations showed the desired fill conditions occur over several tens of microseconds. Taking into account additional delays associated with the puff valve circuit and the noninstantaneous opening of the valve, the range of acceptable delay was consistent with the results of the simulations.

Two complete sets of both magnetic and electric field data were collected in $5 \mathrm{~mm}$ axial increments from 5 to 40 $\mathrm{mm}$ from the coil face. From this data, the velocity of the first sheet was determined to be approximately $4 \mathrm{~cm} / \mu \mathrm{s}$. The production of the plasma sheets was observed to correspond to the half-cycle maximums of the induction coil current as expected. In general, the strongest sheet was produced by the second half cycle. This may have been due to preionization during the first half cycle allowing for strong plasma coupling during the second half cycle. The main body of the plasma was accelerated on the second half cycle of the anode coil current, with a lesser amount accelerated on the third half cycle. Limiting the calculations to those times when the earlier mentioned assumptions are valid, the plasma density of the second half-cycle sheet at $z=7.5 \mathrm{~mm}$ was found to be approximately $10^{16} \mathrm{~cm}^{-3}$. As mentioned earlier, the error bars associated with this calculation are large, possibly one order of magnitude.

In the third set of experiments, the electric and magnetic field data was collected as before with the cathode structure installed and the external insulating magnetic field applied. 
The purpose of these tests was to examine the effect of the external fields on the anode plasma development and movement. The external field had a significant impact on the gas breakdown. The coupling of the pulsed field to the gas appeared much weaker than in the earlier experiments likely resulting in a lower ionization level. The $E$ field probe data was noisier, which complicated the data analysis, increasing the uncertainty in the calculations. The source of this noise has not been determined. However, the plasma behavior recorded was more consistent from shot-to-shot than that collected for earlier experiments. The plasma density was calculated to be of order $10^{15} \mathrm{~cm}^{-3}$ at $z=7.5 \mathrm{~mm}$ with error as large as one order of magnitude.

The anode plasma has been successfully produced at each of three stages of testing and the plasma density appears to be sufficient for the extraction of a beam. In the event that stronger ionization is required, a higher frequency preionization signal may have to be superimposed on the current pulse of the anode coil. ${ }^{9}$

Significant work remains to be completed in order to accurately and completely characterize the CHAMP anode plasma. To better characterize the plasma, a more elaborate instrumentation set is required. In particular, a small array of field probes needs to be assembled and monitored simultaneously during tests to eliminate the error associated with shot-to-shot inconsistencies. If possible, the spacing of the $\dot{B}$ probes on this array should be closer than $5 \mathrm{~mm}$ to better approximate the derivative term in (4). Ideally, an instrument tree could be assembled capable of monitoring the localized plasma density at several distant points simultaneously. Other types of instrumentation must also be considered including interferometric and spectroscopic techniques.

${ }^{1}$ H. Davis, G. Remnev, R. Stinnett, and K. Yatsui, MRS Bull. 21, 58 (1996).

${ }^{2}$ H. Davis et al., Mater. Chem. Phys. 54, 213 (1998).

${ }^{3}$ H. Davis et al., Rev. Sci. Instrum. 68, 332 (1997).

${ }^{4}$ R. Miller, An Introduction to the Physics of Intense Charged Particle Beams (Plenum, New York, 1998).

${ }^{5}$ G. Bird, Molecular Gas Dynamics and the Direct Simulations of Gas Flows (Oxford University Press, New York, 1994).

${ }^{6}$ I. Boyd, J. Comp. Phys. 96, 411 (1991).

${ }^{7}$ I. Hutchinson, Principles of Plasma Diagnostics (Cambridge University Press, Cambridge, 1987).

${ }^{8}$ F. Chen, Introduction to Plasma Physics and Controlled Fusion (Plenum, New York, 1984).

${ }^{9} \mathrm{~T}$. Lockner (personal communication). 